\section{Fondation de la Société médicale du Canton du Jura}

\author{
B. Friolet
}

aventure pour une corporation professionnelle régionale qui, à l'époque, comptait une cinquantaine de membres et fonctionnait plutôt comme une amicale, dont on dit qu'elle se serait même offert le luxe de "renvoyer" son comité pour le fait qu'il n'aurait pas été à même de prévoir un menu à la hauteur des attentes pour la fin d'une séance ... Avec la constitution de sa propre société, la corporation médicale du Canton du Jura mettait un terme à une période d'incertitudes. Elle était désormais détachée de la grande Société médicale du Canton de Berne et pouvait prendre elle-même sa destinée en mains.

\section{Période de transition}

Le 17 mai 1979 est un jour de fête particulier dans les annales de la Fédération des médecins suisses et des institutions du corps médical. C'est en effet ce jour-là, que se déroulèrent les festivités marquant la fondation de la société médicale du nouveau canton, autonome depuis le $1^{\text {er }}$ janvier 1979. Un événement unique, qui va bien au-delà de l'intégration d'une simple organisation professionnelle au sein de la FMH, puisqu'il découle de la création d'un nouveau canton. En ma qualité de président de la Société médicale du Jura-Nord, appuyé par un comité très efficace, il m'appartenait d'organiser cette manifestation. Des délégations de la FMH, de la Société médicale de la Suisse romande et de plusieurs cantons, ainsi que des représentants des autorités jurassiennes prirent part à la cérémonie, dont l'apogée fut la conférence, très remarquée, de la philosophe genevoise Jeanne Hersch, intitulée "Les normes morales face aux progrès de la médecine». Le Bulletin des médecins suisses (No $22 \mathrm{du}$ 30 mai 1979) parlait "d'une fête empreinte de franche amitié, de concorde et d'esprit confraternel». ${ }^{1}$

Ces festivités passées, j’ai assumé la présidence de la nouvelle société durant près de trois ans. La tâche de mener à bien la transition était exaltante, mais très délicate et complexe, laborieuse parfois. Une véritable

\footnotetext{
Je me dois d'ajouter que l'ambiance générale était pesante à ce moment-là. La cérémonie d'accueil très officielle du jeune canton au sein de la Confédération, prévue pour le 11 mai, dont les préparatifs étaient très avancés, avait été annulée sous la pression du groupe Bélier qui invoquait une violente altercation entre le Conseiller national séparatiste Jean-Claude Crevoisier et le conseiller fédéral Kurt Furgler. Une annulation ressentie comme un affront par le peuple Suisse.

Le lendemain du 17 mai, le Rassemblement jurassien faisait paraître dans la presse sous le titre "Société médicale $d u$ Canton $d u$ Jura: débuts boîteux" un article dans lequel il déplorait, entre autres, le manque de panache entourant la cérémonie et le choix de l'oratrice du jour, l'éminente philosophe genevoise Jeanne Hersch, relevant sa réserve notoire à l'égard du séparatisme jurassien. Il exhortait le peuple jurassien à la vigilance à l'occasion de la désignation de responsables de sociétés et associations, me reprochant mes origines alémaniques fribourgeoises et mon appartenance au parti radical, ainsi qu'à la $3^{\mathrm{e}}$ force durant les plébiscites.
}

Correspondance:

Dr Beat Friolet

Rue du Mont-Terri 14

CH-2800 Delémont
La période de transition fut délicate et très intense, car il s'agissait, entre autres, de nous doter des moyens de faire face aux sollicitations d'une administration cantonale enthousiaste, mais encore peu expérimentée, et de veiller à entrer du bon pied dans l'indépendance.

Une longue succession de séances de comité, la mise en place de groupes de réflexion et de travail et des prises de contact aux niveaux les plus divers, furent nécessaires pour mener la tâche à bien. Elles mobilisèrent la quasi totalité des membres de notre société, qui durent faire preuve d'un esprit critique, de pragmatisme et de beaucoup de souplesse. Il y a cependant lieu de relever que, dans ce travail de milice, nous avons heureusement pu bénéficier du soutien et des conseils avisés de la FMH et de nos confrères bernois et romands.

La nouvelle Constitution cantonale - réalisée avec un élan, une compétence et un esprit novateur remarquables - offrait le cadre législatif indispensable à l'exercice de notre profession. Toutefois, en attendant d'avoir trouvé nos propres solutions, la législation bernoise était encore appliquée.

\section{Souveraineté acquise}

Le canton se voulait résolument social et progressiste. On retrouvait partout cette volonté d'être différents, et l'esprit frondeur d'un petit peuple fier de son indépendance et heureux de la liberté acquise. On ressentait, à tous les niveaux, le souci de sortir des sentiers battus et de ne pas simplement devenir "un canton comme les autres". Les groupes d'action et de réflexion, publics et semi-privés, ainsi que des initiatives de tous genres foisonnaient. A titre d'exemple relevons: le planning familial, l'éducation sexuelle à l'école, le premier bureau de la condition féminine créé en Suisse et, plus récemment, l'éligibilité des étrangers dans les parlements communaux. Face à cette effervescence, la jeune société de médecine appelait à une certaine modération, parfois mal perçue. Les différents milieux concernés ont bien dû se rendre à l'évidence que les ressources financières n'étaient pas à la hauteur de leurs aspirations, aussi louables fussent-elles. 
Durant mon mandat, il fallut également freiner le zèle d'autorités communales qui voulaient à tout prix avoir, sur place, leur médecin, leur pharmacie, leur atelier pour handicapés, leur physiothérapeute, leur laboratoire d'analyses médicales et leur classe spéciale. Par ailleurs, des praticiens (médecins, thérapeutes), étrangers pour la plupart, se pressaient au portillon, rêvant d'une terre promise. Une clinique privée proposant des cures biologiques sur les hauteurs des Franches-Montagnes a fermé ses portes à peine installée. Une clinique anglaise du type AMI a heureusement renoncé à ses projets avant de perturber le tissu hospitalier déjà fragile du canton.

\section{Politique du personnel}

$\mathrm{Au}$ niveau des institutions, la politique du personnel constituait, comme ailleurs aussi, une préoccupation majeure. Concilier une enveloppe financière restreinte avec l'exigence de maintenir, voire d'améliorer la qualité d'accueil et des soins, s'avérait difficile et forçait parfois à des compromis. Par souci d'économie, mais aussi en raison du manque de candidatures suisses, les administrations des différents établissements eurent souvent recours à l'engagement de personnel étranger. L'équivalence en termes de formation et de diplômes n'était pas nécessairement garantie et ce personnel éprouvait quelque peine à s'adapter à notre "philosophie» et à nos méthodes de travail. Par ailleurs, du fait de son isolement géographique et de l'absence d'un grand centre urbain, notre région souffrait d'un manque d'attractivité. La relative inexpérience de l'administration qui se mettait en place, l'absence de structures clairement définies, un niveau salarial bas et une fiscalité lourde incitaient plutôt à l'exode des cerveaux.

La médecine transfrontalière, basée sur une convention franco-suisse de 1889 , posait problème à nos confrères d'Ajoie qui se plaignaient d'un manque d'équité et de l'absence de réciprocité dans la prise en charge des prestations par la "Sécurité sociale» française. De nouveaux arrangements ont été trouvés depuis lors.

\section{Propharmacie}

La propharmacie constituait un autre sujet de préoccupation pour notre société. Bien qu'un nombre très limité de confrères exerçaient cette activité, d'âpres discussions eurent lieu entre le corps médical, les pharmaciens et les autorités. Un concept, un peu idéaliste, voire naïf de ma part, visant à une entente cordiale avec nos "cousins" les pharmaciens et qui prévoyait notamment une collaboration dans le domaine de la formation continue, a fait naufrage face aux ailes dures des deux corporations.

\section{Politique hospitalière}

On nous demande occasionnellement quel est notre hôpital cantonal. La réponse est simple, le législateur n'en voulait pas! La commission chargée de la planification hospitalière jugeait le coût d'un tel établissement excessif. Elle considérait que la relative proximité des cliniques universitaires de Bâle, de Berne et accessoirement de Lausanne, avec lesquelles nous entretenions depuis toujours des relations privilégiées, garantissait au patient jurassien l'accès à la médecine de pointe, moyennant des conventions intercantonales. Restait la délicate question du financement des hospitalisations hors canton et les tiraillements avec les assureurs qui en découlent. Les autorités et la commission s'orientaient vers un système de soi-disant complémentarité des trois hôpitaux du canton ou une organisation "multisites». Des solutions difficiles à imposer, car elles se heurtent à des sensibilités et prérogatives locales profondément ancrées. Mais, étant donné l'évolution de la médecine et des coûts qu'elle engendre, le canton devra tôt ou tard trancher et concentrer ses moyens sur un seul site. Délicate décision pour un gouvernement, un parlement et une population "où tout le monde se connaît et se tutoie» et où le politique doit veiller à ménager les susceptibilités.

Ce survol d'une période historiquement unique en Suisse est forcément lacunaire et subjectif. Rétrospectivement je dois avouer que la tâche fut passionnante et que les organes politiques et les différentes organisations professionnelles impliquées dans le processus ont su relever le défi. Aujourd'hui, le corps médical jurassien a complété ses rangs. Que ce soit dans le secteur privé ou hospitalier, il couvre pratiquement toutes les spécialisations et maintient des échanges consultatifs et de formation fructueux avec les facultés de médecine du pays.

Pour ma part, j'ai pu exercer ma profession de pédiatre avec beaucoup de satisfaction et dans une bonne ambiance. J'ai vu le jeune Etat se consolider et s'épanouir tout en évoluant, par la force des choses, vers un canton finalement pas trop différent des autres.

Un membre du Gouvernement relevait récemment que le Jura a aussi de solides atouts: sa souveraineté cantonale, un espace naturel agréable, une économie en voie de modernisation, une main-d'œuvre qualifiée, des établissements scolaires reconnus et une vie socioculturelle intense. Je ne peux que souscrire à ces dires, ayant trouvé dans ce coin de pays, avec ma famille, une terre d'accueil sympathique et une patrie de cœur. 